\title{
In appreciation of Sir Philip Randle: The glucose-fatty acid cycle
}

\author{
Mary C. Sugden* \\ Centre for Diabetes and Metabolic Medicine, Institute of Cell and Molecular Science, Barts and the London School of Medicine \\ and Dentistry, Queen Mary, University of London, 4 Newark Street, Whitechapel, London E1 2AT, UK
}

\begin{abstract}
The coordinated regulation of metabolic fuel selection is crucial to energy homeostasis. Philip Randle and his colleagues developed the fundamental concept of interplay between carbohydrate and lipid fuels in relation to the requirement for energy utilisation and storage. Their insight has fashioned current understanding of the regulation of metabolism in health and disease, as well as providing a springboard for research into the roles of lipid derivatives in insulin resistance and, at the transcriptional level, lipid-regulated nuclear hormone receptors.
\end{abstract}

Glucose: Lipid: Insulin: Signalling: Insulin resistance: Nuclear hormone receptors: Peroxisome proliferator-activated receptors: Type 2 diabetes mellitus

As I write this appreciation of Sir Philip Randle, who died aged 80 on 26 September 2006, the imprinting of Philip's (great) presence and personality has become increasingly obvious. Many of us owe a tremendous debt to Philip, and I feel privileged to write this appreciation of his work. This research article is primarily concerned with just one of Philip's many contributions, data published by Randle, Garland, Hales and Newsholme in the Lancet in 1963 (Randle et al. 1963) entitled 'The glucose-fatty acid cycle: its role in insulin sensitivity and the metabolic disturbances of diabetes mellitus'. Its origin encapsulates the synergistic cooperation and team spirit that Philip fostered. This paper, one of the best-known of Philip's papers, laid down the fundamental concept of reciprocal substrate competition between glucose and non-esterified fatty acids (NEFA) (the major fuels that are oxidised to provide ATP in mammals) in normal physiology in muscle, but it has proved to be applicable to a range of other oxidative tissues. The authors proposed that: 'The cycle ... provides a primitive mechanism which, quite independently of hormonal control, will tend to maintain a constant plasma glucose concentration in animals that feed intermittently', emphasising the role of its operation in health. Importantly, it was also proposed that the origin of impaired glucose utilisation in diabetes mellitus, then viewed primarily as a disorder of carbohydrate metabolism, might in part reflect excessive release of NEFA from adipose tissue triacylglycerol. This research article also predicted that lipid derivatives might be the active species in mediating insulin resistance in skeletal muscle: 'Growth hormone, corticosteroids and adrenaline ... by accelerating release of fatty acids from ... muscle glycerides ...may through this action inhibit uptake of glucose by muscle at a particular insulin concentration - i.e., induce insulin insensitivity'. Thus the glucose-fatty acid (FA) cycle has resonance not only for diabetes but also other metabolic diseases associated with insulin resistance, including obesity. Philip, completing the full circle of his contributions, reviewed the current concept of regulatory interactions between lipids and carbohydrates 35 years later, in his last review and publication (Randle, 1998).

\section{Substrate competition and the glucose-fatty acid cycle}

The reciprocal cross-talk between glucose and FA and the coordinate control of the utilisation of these primary oxidative fuels is vitally important for whole-body nutrient homeostasis. In the paper of 1963, which warrants the description of landmark, Randle and his co-workers highlighted the ability of oxidative muscle (red skeletal muscle and heart), to alternate between the use of lipid fuels (albumin-bound NEFA and the ketone bodies) and glucose for ATP production, depending on their relative availabilities. The operation of the glucoseFA cycle in skeletal muscle in particular is of major importance for whole-body nutrient homeostasis in the intact animal, both because of the major drain that the skeletal muscle mass imposes on available nutrients, even in the resting state, and because skeletal muscle is a major site of insulin-stimulated glucose disposal and thus a major site of lipid-induced insulin resistance.

The first major tenet of the glucose-FA cycle, as described in the 1963 Lancet article, is that the increased provision of exogenous lipid fuels or the increased breakdown of endogenous triacylglycerol promotes the use of FA (and their partial oxidation products, the ketone bodies) as oxidative fuels and, in so doing, glucose utilisation is blocked. The primary site of exogenous production of NEFA under conditions of metabolic stress (increased cardiac workload, skeletal muscle exercise); hormonal stress (e.g. excess glucocorticoids, insulin deficiency) or carbohydrate stress (starvation) was considered to be adipose tissue, as had been demonstrated by measuring plasma

Abbreviation: FA, fatty acid.

* Corresponding author: Professor Mary C. Sugden, fax 0207882 2186, email m.c.sugden@qmul.ac.uk 
concentrations of FA and release of FA from rodent adipose tissue in vivo. The experimental approach used was straightforward, involving measurement of glycerol release from isolated tissues (heart, skeletal muscle, fat pads) as an index of endogenous triacylglerol breakdown. It was observed that breakdown of endogenous (stored) triacylglycerol in isolated rat heart and skeletal muscle (diaphragm) was augmented by antecedent starvation, alloxan-diabetes or treatment with growth hormone or cortisol in vivo, or by treatment of isolated muscles with growth hormone or adrenaline in vitro. In contrast, glycerol output by adipose tissue was suppressed by insulin. Secondly, and most importantly, it was shown that exogenous provision of lipid fuels (albumin-bound FA, short-chain FA, ketone bodies) in the presence or absence of added insulin suppressed glucose uptake and glycolysis to levels that had been observed when tissues were obtained from diabetic animals. Previous research from the group had shown that the same manoeuvre suppressed pyruvate oxidation (Garland et al. 1962). Subsequently a succession of papers from this and other groups has provided evidence of the biochemical mechanisms that might be involved (for review, see Holness et al. 1997; Randle, 1998; Muoio \& Newgard, 2006). Philip himself developed a lasting interest in the regulation of the pyruvate dehydrogenase complex, which he pursued to great effect at Bristol and subsequently at Oxford.

The Cori's had previously introduced the concept that intertissue fluxes of glucose and the glycolytic derivative lactate were important for maintaining the level of glycaemia (Cori cycle). Philip and his colleagues took this one step further, in that they introduced the idea that the provision of FA mobilised from stored triacylglycerol in adipose tissue, or alternatively within the muscle itself, could co-ordinately block the use of another substrate, glucose, of which the stores were limited but which is an obligatory energy substrate for some tissues. On the basis of their findings that when hearts were perfused with FA and ketone bodies, the glycolytic rate was decreased but the combined output of lactate and pyruvate was not diminished, and that in spite of impaired glucose phosphorylation, glucose 6-phosphate and fructose 6-phosphate accumulate, Randle and colleagues suggested that the degree of impairment of glucose degradation at four key steps (membrane transport of glucose, glucose phosphorylation, the phosphofructokinase reaction and the pyruvate dehydrogenase reaction) was graded, most severe at the level of the pyruvate dehydrogenase reaction: 'This could allow glycolysis to continue its function as an important pathway for the synthesis of cell constituents during periods of carbohydrate deprivation while ensuring that the end products of glycolysis, pyruvate and lactate, are not oxidised but reconverted to glucose in the liver'. This paper therefore indicated the biochemical mechanism that could underlie the operation of the Cori cycle, and this was subsequently verified in vivo (see e.g. Holness et al. 1997). Studies in man up to 1998 describing the effects of FA on glucose metabolism in vivo are detailed in Philip's 1998 review (Randle, 1998) and by Keith Frayn (Frayn, 2003) as part of a Biochemical Society Symposium held in Philip's honour in 2003.

In addition, and of major importance, the authors made it clear that the relationship between glucose and FA metabolism is reciprocal, and not dependent. Thus, when glucose is abundant, glucose utilisation can block FA release (and therefore oxidation): 'In the blood phase, uptake of glucose by adipose tissue is depicted as inhibiting the flow of fatty acids from adipose tissue (and of ketone bodies formed from them in the liver) to muscle through the blood stream'. This theme was developed at the intracellular level by McGarry and colleagues (for review, see McGarry, 1998), who demonstrated that high glucose concentrations suppressed hepatic FA oxidation (predominantly ketogenesis) via malonyl-CoA-mediated inhibition of carnitine palmitoyltransferase 1, thereby promoting partitioning of incoming NEFA from adipose tissue away from oxidation towards (re-)esterification. It is now recognised that, although muscle is not a conventionally 'lipogenic' tissue like liver, this same overall mechanism operates both in heart and skeletal muscle, tissues that contain a different isoform of acetylCoA carboxylase (which catalyses the conversion of cytoplasmic acetyl-CoA to malonyl-CoA) from that expressed in the liver (for review, see Zammit, 1999).

Any mismatch between FA uptake into muscle and the requirement for FA oxidation for ATP production, when occurring under conditions where glucose conversion to malonyl-CoA is rapid, will result in intramyocyte triacylglycerol accumulation. This concept has been developed most extensively within the context of the aetiology of muscle insulin resistance (see later). Operating in the reverse direction (again a cycle), lowering of malonyl-CoA levels will allow the breakdown of intramyocellular triacylglycerol as a supplementary fuel, as was observed by Randle and colleagues as part of their studies described in the Lancet article.

\section{The role of insulin}

In the Lancet article, it was stated by Philip and his coworkers that: 'Control by the cycle is modified by insulin, which enhances glucose uptake in muscle and adipose tissue, inhibits release of fatty acids in adipose tissue, and increases esterification of fatty acids in adipose tissue and muscle'. Thus, the action of insulin to suppress FA release from adipose tissue forces muscle towards the use of glucose. Data from Frayn's group have demonstrated that after isoenergetic meals of differing macronutrient composition, the carbohydrate and fat balances change reciprocally in accordance with meal composition, such that total energy balance is unchanged (Frayn, 2003). In recent years, it has become apparent that there is an additional interlinked inter-organ cycle, by which lipids can influence insulin secretion. In the short term, FA stimulate insulin secretion in a glucose-dependent manner and can potentiate glucose stimulated insulin secretion via as yet undefined lipid signalling molecules (Prentki et al. 1992; for review, see Sugden \& Holness, 2004). Recent research has also implicated a cell-surface G-protein coupled receptor (GPR), GPR40, which may serve to mediate direct effects of FA on glucose-stimulated insulin secretion by modulating the activity of voltage gated $\mathrm{K}^{+}$channels (for review, see Gromada, 2006). Small molecule GPR40 agonists are currently considered as potential treatments for type 2 diabetes. However, the long-term exposure of pancreatic islets to high levels of FA is detrimental to islet function, particularly in conjunction with chronic exposure to high glucose. This phenomenon, referred to as glucolipotoxicity, both highlights the requirement for integrated regulation of 
carbohydrate and fat metabolism and indicates one of the deleterious sequels of a malfunction of the glucose-FA cycle (Poitout \& Robertson, 2002).

\section{A mismatch between glucose and lipid supply, formation and usage leads to insulin resistance}

A second component of the glucose-FA cycle was the idea that the oxidation of lipid fuels could underlie the development of insulin resistance. Thus, in their final summary of their Lancet paper, Randle and colleagues suggested: 'that there is a distinct biochemical syndrome ... due to the breakdown of glycerides in adipose tissue and muscle, the symptoms of which are a high concentration of plasma non-esterified fatty acids, impaired sensitivity to insulin, ... and, frequently, impaired glucose tolerance.' This was a strategically important concept since hitherto it had been considered that diabetes was primarily a disorder of carbohydrate metabolism. Many studies have shown an association between skeletal muscle insulin resistance and intramyocellular lipid accumulation: it is still uncertain whether this is a cause or effect of insulin resistance (for review, see Sugden \& Holness, 2006). However, in animal models in which the tissue triacylglycerol content is depleted by adenoviral expression of the adipokine leptin, there is a close inverse relationship between whole-body insulin sensitivity and muscle and liver triacylglycerol content (Koyama et al. 1997), elevation of muscle malonyl-CoA concentrations in several models of rodent obesity occur in conjunction with increased intramyocellular triacylglycerol and accumulation of long-chain acyl-CoA (for review, see Ruderman et al. 2003; Saha \& Ruderman, 2003), and mice lacking the muscle acetyl-CoA carboxylase isoform are protected against diet-induced insulin resistance (Abu-Elheiga et al. 2001). As noted earlier, Randle and colleagues observed increased glycerol production by hearts from starved and diabetic rats: this is consistent with either greater intramyocellular triacylglycerol storage or more labile triacylglycerol stores. It is emerging that intramyocellular triacylglycerol accumulation per se is not responsible for reduced skeletal muscle insulin action, but is a surrogate marker of other lipid intermediates known to suppress insulin sensitivity: these include long-chain acyl-CoA (for review, see Ellis et al. 2000; Thompson \& Cooney, 2000; Hegarty et al. 2003) which may be derived from either circulating FA or FA released from intramyocellular triacylglycerol, $\beta-\mathrm{OH}$-butyrylcarnitine (for review, see Muoio \& Newgard, 2006) and ceramide, a sphingolipid derived from the condensation of palmitoyl-CoA and serine (for review, see Summers \& Nelson, 2005). Long-chain acyl-CoA can alter insulin action by impairing signalling via the insulin receptor and insulin receptor substrate-1 via activation of a serine kinase cascade, culminating in serine phosphorylation of the insulin receptor. The FA signalling cascade may involve an inhibitor of $\kappa \mathrm{B}$ kinase. Inhibition of the inhibitor of $\kappa \mathrm{B}$ kinase activity by salicylates suppresses activation of the NF- $\kappa \mathrm{B}$ transcription factor and prevents the development of skeletal-muscle insulin resistance (Kim et al. 2001; Perseghin et al. 2003) and overexpression of a constitutively active version of the NF- $\kappa$ B activating kinase results in insulin resistance and diabetes (Cai et al. 2005). In the Lancet article, it was noted that sodium salicylate could abolish the inhibitory effects of FA and ketone bodies on diminished phosphorylation of glucose and impaired glycolysis in muscle and it was suggested that: 'This effect of salicylate could be an important factor in its known hypoglycaemic action in human diabetes'.

It follows from Randle's work that an inability to regulate glycaemia (e.g., because of inappropriately high rates of hepatic glucose production due to hepatic insulin resistance) impinges on lipid handling by skeletal muscle. There are several mechanisms by which this may be achieved, in addition to acute effects of high glucose to increase the rate of malonyl-CoA production. It is now known that prolonged hyperglycaemia upregulates the lipogenic transcription factor sterol-regulatory-element-binding-protein-1c as well as key genes encoding lipogenic enzymes, permitting an increased capacity for rates of FA synthesis de novo (for review, see Eberle et al. 2004). Sterol-regulatory-element-binding-protein-1c and its target enzymes are expressed at higher levels in tissues of 'insulin resistant' obese mice which have defective receptors for the leptin. Parenthetically, the new data implicating adipose-derived factors such as leptin and adiponectin in the modulation of glucose control in muscle, if it were to occur in association with effects of muscle-derived factors on adipose tissue, would extend the concept of regulatory interorgan interplay for the control of fuel homeostasis utilising the 'blood phase'.

\section{Expanding the glucose-fatty acid cycle to gene expression}

As well as their interaction with GPR40, lipids (including long chain FA) can exert major effects at the level of gene expression. The PPARs $(\alpha, \beta / \delta$ and $\gamma)$ mediate adaptive metabolic responses to increased systemic lipid availability following activation by binding of naturally occurring endogenous or dietary FA or lipid derivatives (for review, see Desvergne et al. 2006). The PPAR $\alpha$ and PPAR $\delta$ isoforms are enriched in tissues which have a high capacity for FA oxidation, including liver and muscle: ligand binding activates a programme of lipid-induced activation of genes encoding proteins involved in FA uptake, activation and $\beta$-oxidation. Tissues of $\operatorname{PPAR} \alpha$ null mice become lipid engorged on starvation and after high-fat feeding (Leone et al. 1999; Kersten et al. 1999). Excessive activation of PPAR $\alpha$ in the heart drives myocardial FA utilisation and leads to impaired glucose uptake and utilisation and importantly, PPAR $\alpha$ mediated cross talk may comprise a transcriptional equivalent of the glucose-FA cycle (for review, see Finck, 2006). The precise identity of natural endogenous $\operatorname{PPAR} \alpha$ ligands remains unclear. In liver, newly-synthesised fat or dietary fat (but not pre-existing fat) may function as endogenous PPAR $\alpha$ activators (Chakravarthy et al. 2005). Should this also occur in muscle, enhanced lipogenic flux in muscle may lead to increased accumulation of cellular lipids that act as PPAR ligands, re-establishing a competitive advantage of FA over glucose as oxidative substrate. PPAR $\gamma$ is found in adipose tissue, where its activation promotes lipid uptake, synthesis and storage. By draining FA from the circulation into storage as adipose tissue triacylglycerol, PPAR $\gamma$ activation influences substrate competition between FA and glucose in muscle such that glucose oxidation by muscle is favoured (for review, see Frayn, 2002). 


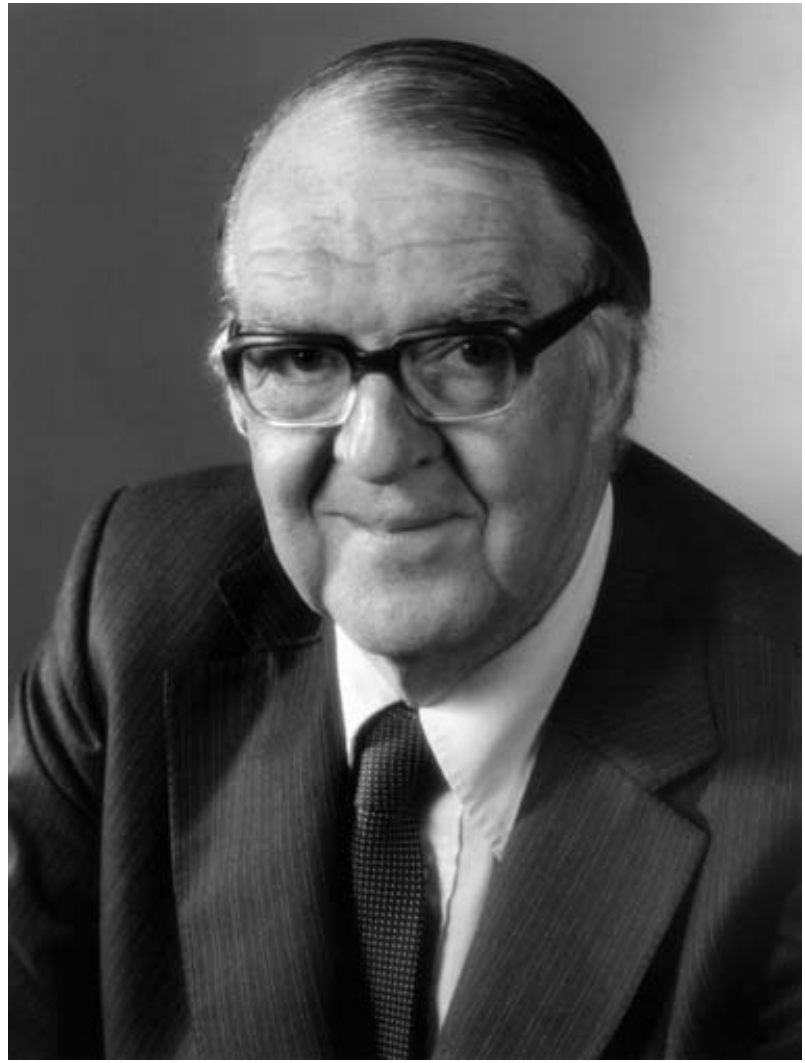

Professor Sir Philip Randle, MA, PhD, MD, FRCP, FMedSci, FRS (C) The Godfrey Argent Studio).

\section{Epilogue}

It is now evident that lipids or their derivatives not only compete with glucose as an oxidative substrate, but can also influence insulin secretion and insulin signalling. The importance of appropriate control of lipid availability, storage and oxidation and of insulin secretion has been the subject of investigation of many of Philip's close colleagues and friends from his Cambridge and Bristol years, as well as his later years at Oxford, together with internationally. In writing this appreciation, I have tried where possible to refer to excellent reviews on recent research emanating from Philip's original hypothesis area which are referenced more comprehensively. Philip will be a hard act to follow and he will be greatly missed.

\section{Acknowledgements}

Thanks for Philip, Eric and Peter for their insight and to Philip, and friends and colleagues met through Philip, for making working in this area so much fun.

\section{References}

Abu-Elheiga L, Matzuk MM, Abo-Hashema KA \& Wakil SJ (2001) Continuous fatty acid oxidation and reduced fat storage in mice lacking acetyl-CoA carboxylase 2. Science 291, 2613-2616.

Cai D, Yuan M, Frantz DF, Melendez PA, Hansen L, Lee J \& Shoelson SE (2005) Local and systemic insulin resistance resulting from hepatic activation of IKK-beta and NF-kappaB. Nat Med 11, 183-190.

Chakravarthy MV, Pan Z, Zhu Y, Tordjman K, Schneider JG, Coleman T, Turk J \& Semenkovich CF (2005) 'New' hepatic fat activates PPARalpha to maintain glucose, lipid, and cholesterol homeostasis. Cell Metab 1, 309-322.

Desvergne B, Michalik L \& Wahli W (2006) Transcriptional regulation of metabolism. Physiol Rev 86, 465-514.

Eberle D, Hegarty B, Bossard P, Ferre P \& Foufelle F (2004) SREBP transcription factors: master regulators of lipid homeostasis. Biochimie 86, 839-848.

Ellis BA, Poynten A, Lowy AJ, Furler SM, Chisholm DJ, Kraegen EW \& Cooney GJ (2000) Long-chain acyl-CoA esters as indicators of lipid metabolism and insulin sensitivity in rat and human muscle. Am J Physiol Endocrinol Metab 279, E554-E560.

Finck BN (2006) Effects of PPARalpha on cardiac glucose metabolism: a transcriptional equivalent of the glucose-fatty acid cycle? Expert Rev Cardiovasc Ther 4, 161-171.

Frayn KN (2002) Adipose tissue as a buffer for daily lipid flux. Diabetologia 45, 1201-1210.

Frayn KN (2003) The glucose-fatty acid cycle: a physiological perspective. Biochem Soc Trans 31, 1115-1119.

Garland PB, Newsholme EA \& Randle PJ (1962) Effect of fatty acids, ketone bodies, diabetes and starvation on pyruvate metabolism in rat heart and diaphragm muscle. Nature 195, $381-383$.

Gromada J (2006) The free fatty acid receptor GPR40 generates excitement in pancreatic beta-cells. Endocrinology 147, 672-673.

Hegarty BD, Furler SM, Ye J, Cooney GJ \& Kraegen EW (2003) The role of intramuscular lipid in insulin resistance. Acta Physiol Scand 178, $373-383$.

Holness MJ, Fryer LG \& Sugden MC (1997) Endocrine and nutritional modulation of glucose disposal and storage in muscle. Biochem Soc Trans 25, 1-7.

Kersten S, Seydoux J, Peters JM, Gonzalez FJ, Desvergne B \& Wahli W (1999) Peroxisome proliferator-activated receptor alpha mediates the adaptive response to fasting. J Clin Invest 103, 1489-1498.

Kim JK, Kim YJ, Fillmore JJ, Chen Y, Moore I, Lee J, Yuan M, Li ZW, Karin M, Perret P, Shoelson SE \& Shulman GI (2001) Prevention of fat-induced insulin resistance by salicylate. J Clin Invest 108, 437-446.

Koyama K, Chen G, Lee Y \& Unger RH (1997) Tissue triglycerides, insulin resistance, and insulin production: implications for hyperinsulinemia of obesity. Am J Physiol 273, E708-E713.

Leone TC, Weinheimer CJ \& Kelly DP (1999) A critical role for the peroxisome proliferator-activated receptor alpha (PPARalpha) in the cellular fasting response: the PPARalpha-null mouse as a model of fatty acid oxidation disorders. Proc Natl Acad Sci USA 96, 7473-7478.

McGarry JD (1998) Glucose-fatty acid interactions in health and disease. Am J Clin Nutr 67, 500S-504S.

Muoio DM \& Newgard CB (2006) Obesity-related derangements in metabolic regulation. Апnи Rev Biochem 75, 367-401.

Perseghin G, Petersen K \& Shulman GI (2003) Cellular mechanism of insulin resistance: potential links with inflammation. Int $J$ Obes Relat Metab Disord 27, Suppl. 3, S6-S11.

Poitout V \& Robertson RP (2002) Minireview: Secondary beta-cell failure in type 2 diabetes-a convergence of glucotoxicity and lipotoxicity. Endocrinology 143, 339-342.

Prentki M, Vischer S, Glennon MC, Regazzi R, Deeney JT \& Corkey BE (1992) Malonyl-CoA and long chain acyl-CoA esters as metabolic coupling factors in nutrient-induced insulin secretion. $J$ Biol Chem 267, 5802-5810.

Randle PJ (1998) Regulatory interactions between lipids and carbohydrates: the glucose fatty acid cycle after 35 years. Diabetes Metab Rev 14, 263-283. 
Randle PJ, Garland PB, Hales CN \& Newsholme EA (1963) The glucose fatty-acid cycle. Its role in insulin sensitivity and the metabolic disturbances of diabetes mellitus. Lancet 1, 785-789.

Ruderman NB, Saha AK \& Kraegen EW (2003) Minireview: malonyl CoA, AMP-activated protein kinase, and adiposity. Endocrinology 144, 5166-5171.

Saha AK \& Ruderman NB (2003) Malonyl-CoA and AMP-activated protein kinase: an expanding partnership. Mol Cell Biochem 253, $65-70$.

Sugden MC \& Holness MJ (2004) Potential role of peroxisome proliferator-activated receptor-alpha in the modulation of glucosestimulated insulin secretion. Diabetes 53, Suppl. 1, S71-S81.
Sugden MC \& Holness MJ (2006) Skeletal muscle lipid metabolism and the adipo-muscular axis. Future Lipidology 1, $153-162$.

Summers SA \& Nelson DH (2005) A role for sphingolipids in producing the common features of type 2 diabetes, metabolic syndrome X, and Cushing's syndrome. Diabetes 54, 591-602.

Thompson AL \& Cooney GJ (2000) Acyl-CoA inhibition of hexokinase in rat and human skeletal muscle is a potential mechanism of lipid-induced insulin resistance. Diabetes 49, 1761-1765.

Zammit VA (1999) The malonyl-CoA-long-chain acyl-CoA axis in the maintenance of mammalian cell function. Biochem $J \mathbf{3 4 3}$, $505-515$ 